\title{
Integration of single-fiber reflectance spectroscopy into ultrasound-guided endoscopic lung cancer staging of mediastinal lymph nodes
}

\author{
Stephen Chad Kanick \\ Erasmus Medical Center \\ Center for Optical Diagnostics and Therapy \\ Department of Radiation Oncology \\ Rotterdam 3015 GE \\ The Netherlands
}

\section{Cor van der Leest}

Joachim G. J. V. Aerts

Amphia Hospital

Department of Pulmonary Diseases

Breda, The Netherlands and

Erasmus Medical Center

Department of Pulmonary Diseases

Rotterdam 3000 CA

The Netherlands

Henk C. Hoogsteden

Erasmus Medical Center

Department of Pulmonary Diseases

Rotterdam 3000 CA

The Netherlands

\section{Slávka Kaščáková}

Henricus J. C. M. Sterenborg

Erasmus Medical Center

Center for Optical Diagnostics and Therapy

Department of Radiation Oncology

Rotterdam 3015 GE

The Netherlands

\author{
Arjen Amelink \\ Erasmus Medical Center \\ Center for Optical Diagnostics and Therapy \\ Department of Radiation Oncology \\ Rotterdam 3015 GE \\ The Netherlands \\ and \\ Erasmus Medical Center \\ Department of Pulmonary Diseases \\ Rotterdam 3000 CA \\ The Netherlands
}

\begin{abstract}
We describe the incorporation of a single-fiber reflectance spectroscopy probe into the endoscopic ultrasound fine-needle aspiration (EUS-FNA) procedure utilized for lung cancer staging. A mathematical model is developed to extract information about the physiological and morphological properties of lymph tissue from singlefiber reflectance spectra, e.g., microvascular saturation, blood volume fraction, bilirubin concentration, average vessel diameter, and Mie slope. Model analysis of data from a clinical pilot study shows that the single-fiber reflectance measurement is capable of detecting differences in the physiology between normal and metastatic lymph nodes. Moreover, the clinical data show that probe manipulation within the lymph node can perturb the in vivo environment, a concern that must be carefully considered when developing a sampling strategy. The data show the feasibility of this novel technique; however, the potential clinical utility has yet to be determined. ๑ 2010 Society of Photo-Optical Instrumentation Engineers. [DOI: 10.1117/1.3290822]
\end{abstract}

Keywords: tissue diagnostics; biomedical optics; fiber optic applications; medicine; spectroscopy.

Paper 09383R received Aug. 28, 2009; revised manuscript received Oct. 20, 2009; accepted for publication Nov. 17, 2009; published online Jan. 15, 2010.

\section{Introduction}

Lung cancer causes the most cancer-related deaths in both men and women worldwide. ${ }^{1}$ For patients diagnosed with limited stage non-small-cell lung cancer (NSCLC), treatment options are dependent on the presence of metastatic cancer in the mediastinal lymph nodes. ${ }^{2-4}$ For patients diagnosed with

Address all correspondence to: Stephen Chad Kanick, Erasmus Medical Center, Center for Optical Diagnostics and Therapy, Department of Radiation Oncology, Rotterdam 3015 GE, The Netherlands. Tel: 31-10-7032102; E-mail: s.kanick@erasmusmc.nl
NSCLC, treatment in the absence of metastatic cancer involves surgical resection of the tumor; however, in the presence of metastatic cancer, the treatment of choice is systemic chemotherapy combined with radiotherapy. ${ }^{2}$ Following diagnosis of NSCLC, the mediastinal lymph nodes are imaged, usually with a computed tomography (CT) scan and/or positron emission tomography (PET) scanning, but the specificity of these imaging modalities are not reliable enough to properly diagnose cancer, and histopathological examination of

1083-3668/2010/15(1)/017004/8/\$25.00 @ 2010 SPIE 


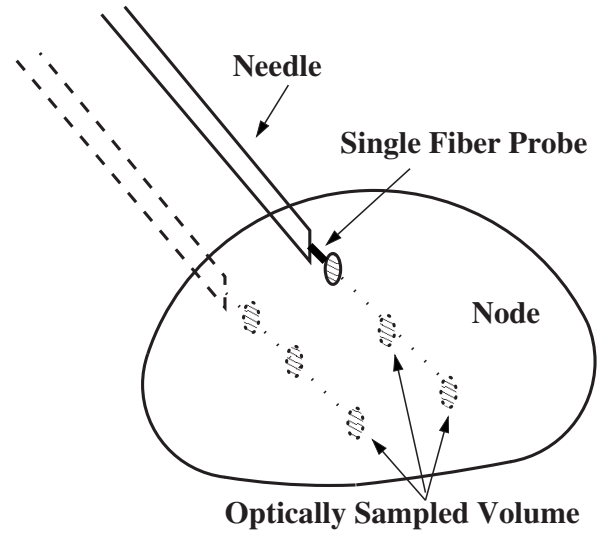

Fig. 1 Schematic of proposed sampling strategy.

any enlarged lymph node is required. The gold standard procedure for mediastinal staging is surgical biopsy of the lymph node $^{2}$ (termed mediastinoscopy). A novel alternative method to stage lymph nodes is the endoscopic ultrasound fine-needle aspiration (EUS-FNA), which utilizes an ultrasound probe to visualize the lymph node being sampled, offering the clinician access to mediastinal lymph nodes with lower costs and less patient morbidity than mediastinoscopy. ${ }^{2-4}$ However, EUSFNA has a low sensitivity and is associated ${ }^{2}$ with a false negative (FN) rate of $\approx 23 \%$ (compared with the $9 \% \mathrm{FN}$ rate of mediastinoscopy). The high EUS-FNA FN rate is attributable to localized malignant areas within the node that were not sampled during the biopsy. This complication necessitates the sampling of multiple sites within the node or requires the presence of a cytologist on-site. On average, five sampling sites within the node are biopsied during EUS-FNA, a total that cannot be easily increased because more samples would require more punctures into the node and longer procedure times; factors that would negatively affect patient comfort.

Incorporation of a fiber optic reflectance device into the EUS-FNA procedure may assist in the identification of malignant regions within the lymph nodes during biopsy, potentially reducing the FN rate of the current procedure. The fiber optic device would enable rapid optical sampling from multiple local tissue regions throughout the lymph node, with the optical properties accurately estimated within small volumes $\left(\approx 0.1 \mathrm{~mm}^{3}\right)$ of tissue optically interrogated during measurement. The schematic in Fig. 1 shows a proposed sampling strategy for EUS-FNA with the incorporated optical device, with the optical fiber sampling multiple $(\approx 10$ to 50$)$ samples acquired within the same time required for one biopsy acquisition in the current procedure. This strategy would result in characterization of a larger volume of the lymph node than is achieved with the current procedure, reducing the chance that malignant areas are not biopsied.

Reflectance spectra measured in the UV-VIS wavelength range contain information about tissue absorption and scattering properties; ${ }^{5}$ information that can be used ${ }^{6-8}$ to describe tissue physiology (e.g., vascular oxygen saturation, blood volume fraction) and morphology (e.g., tissue/cell/organelle size and density), and can be used to differentiate between normal and malignant tissue. Classical reflectance spectroscopic devices that utilize multiple optical fibers to deliver and collect light during measurement ${ }^{6-8}$ are too large to fit through the EUS-FNA biopsy needle (with an inner diameter of $\approx 460 \mu \mathrm{m}$ ). This application requires the use of a single optical fiber with fiber diameters in the range of 200 to $400 \mu \mathrm{m}$ that could be incorporated into the narrow channel of the EUS-FNA device.

The concept of single-fiber (SF) reflectance spectroscopy has been previously proposed, ${ }^{9,10}$ but these studies were limited to qualitative analysis of spectra because they lacked an accurate description of the relationship between the photon path length and the tissue optical properties. Recently, our group developed a novel empirical relationship between the SF photon path length and both the absorption coefficient and reduced scattering coefficient within an optically sampled turbid medium. ${ }^{11}$ This advancement enables quantitative analysis of SF reflectance spectra measured in the UV-VIS wavelength range that provides information about the wavelengthdependent optical properties and enables quantitative extraction of tissue physiological and morphological parameters.

The aims of this study are to investigate the feasibility and identify the technical challenges associated with incorporating an SF spectroscopy device into the EUS-FNA procedure for lung cancer staging. A mathematical model is developed to analytically describe the SF reflectance spectra measured in lymph node tissue in vivo; work that requires characterization of the basis set of chromophores in lymph tissue. The model is applied to a set of clinical pilot data and used to extract physiological and morphological parameters from the SF reflectance spectra measured in normal and metastatic lymph nodes. This paper also presents the practical and technical challenges associated with the novel technique.

\section{Methods}

\subsection{Clinical Study}

Recently, we conducted a clinical pilot study to utilize the combined EUS-FNA with incorporated SF spectroscopy technique during a staging procedure for lung cancer, with data collected at the Erasmus Medical Center and Amphia hospitals (both located in the Netherlands). The experimental protocol was approved by the Institutional Review Board (IRB) at both locations. Data acquired from an initial patient enrolled within the study were used to develop a mathematical model for analysis of the lymph node spectra; in that case, multiple spectra were acquired from a single lymph node that was subsequently determined to contain metastatic cancer by cytology. The mathematical model was then applied to data acquired from 12 patients enrolled in the clinical pilot study, with multiple spectra recorded from seven normal nodes and eight nodes containing metastatic cancer as confirmed by cytology (throughout this paper lymph nodes containing metastatic cancer are referred to as metastatic lymph nodes). Each node was sampled with a new sterile SF reflectance probe. SF measurements were performed with the SF probe extended through the EUS-FNA needle and allowed to rest with no external pressure applied to the probe during spectral acquisition. Previously it has been shown that pressure exerted by a fiber optic probe on tissue during measurements can have effects on the tissue physiology. ${ }^{12}$ This study considers the effects of probe pressure on the measured physiological param- 


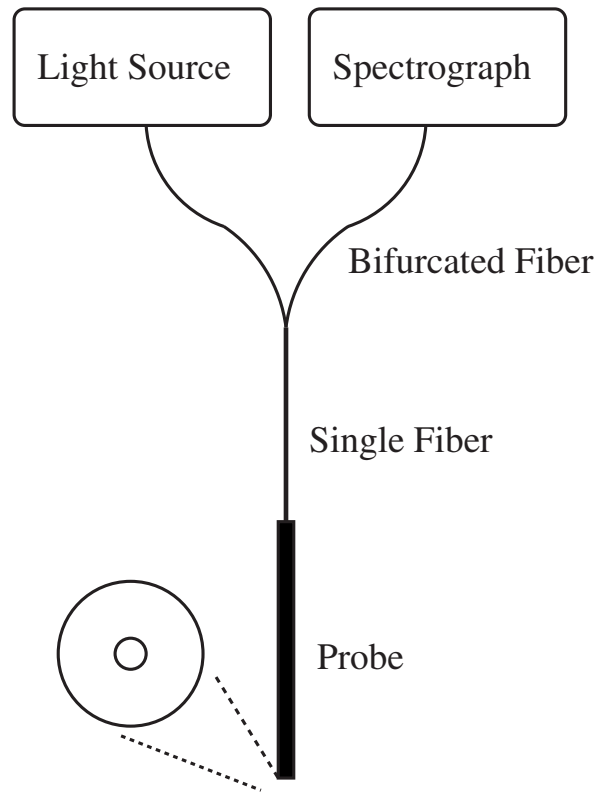

Fig. 2 Schematic of SF reflectance probe machinery.

eters by performing a subset of measurements on two lymph nodes while pressure was applied to the SF probe tip during measurement.

The experimental setup utilized here has been described in detail previously. ${ }^{11}$ In brief, the setup utilized a single optical fiber (internal core diameter of $320 \mu \mathrm{m}$ ) connected to a bifurcated fiber with one arm leading from a halogen light source (HL-2000-FHSA; Ocean Optics; Duiven, the Netherlands) and the second arm leading to a spectrophotometer (SD 2000; Ocean Optics; Duiven, the Netherlands). Figure 2 shows a schematic of the fiber probe. Spectral reflections at the probe tip due to the refractive index mismatch between fiber and sample are minimized by polishing the SF probe tip at an angle of $15 \mathrm{deg}$. A calibration procedure was described previously ${ }^{11}$ to account for other internal reflections, variability in lamp-specific output, and in fiber-specific transmission properties.

\subsection{Mathematical Model of SF Reflectance Spectra}

SF reflectance spectra $\left(\mathbf{R}_{\mathrm{SF}}\right)$ were analyzed using an analytical model to describe the wavelength-dependent optical properties, and in turn, extract physiological and morphological information (throughout this manuscript, terms shown in bold are wavelength dependent). Previously, a similar model was utilized $^{6-8}$ for analysis of other tissues (e.g., lung, breast, and oral mucosa), and is given as

$$
\mathbf{R}_{\mathrm{SF}}=\left[a_{1}\left(\frac{\lambda}{\lambda_{0}}\right)^{a_{2}}+a_{3}\left(\frac{\lambda}{\lambda_{0}}\right)^{-4}\right] e^{-\mu_{\mathbf{a}}^{\text {tissue }}\left\langle\mathbf{L}_{\mathbf{S F}}\right\rangle} .
$$

Here, the term within the square brackets is the background scattering model that is a combination of Mie and Rayleigh scattering, given by wavelength-dependent power law functions with fitted parameters specifying the Mie amplitude $\left(a_{1}\right)$, Mie slope $\left(a_{2}\right)$, and Rayleigh amplitude ${ }^{13}\left(a_{3}\right)$. Attenuation due to absorption within the tissue is modeled using the modified Beer-Lambert law and is a function of both the tis- sue absorption coefficient $\left(\mu_{\mathrm{a}}^{\text {tissue }}\right)$ and the SF photon path length $\left(\left\langle\mathbf{L}_{\mathbf{S F}}\right\rangle\right)$. The basis set of chromophores in lymph tissue is not well characterized, therefore, our initial model assumed that absorption was attributable to oxygenated and deoxygenated hemoglobin $\left(\mathrm{HbO}_{2}\right.$ and $\mathrm{Hb}$, respectively) within the local microvasculature $\left(\mu_{\mathbf{a}}^{\text {vasc }}\right)$, and is given in Ref. 6 as

$$
\mu_{\mathrm{a}}^{\text {tissue }}=\mu_{\mathrm{a}}^{\text {vasc }}=\rho \mathbf{C}_{\mathbf{v}}\left[\mathrm{StO}_{2} \mu_{\mathrm{a}}^{\mathrm{HbO}_{2}}+\left(1-\mathrm{StO}_{2}\right) \mu_{\mathrm{a}}^{\mathrm{Hb}}\right] .
$$

Here, $\rho$ is the blood volume fraction, $\mathrm{StO}_{2}$ is the microvascular hemoglobin oxygen saturation, $\mathbf{C}_{\mathbf{v}}$ is a factor that accounts for the effect of discrete blood vessels on the absorption coefficient and enables estimation of the average vessel diameter $d_{v}$. Previously, ${ }^{11}$ we showed that the photon path length is dependent on $\mu_{\mathbf{a}}$, the reduced scattering coefficient $\left(\mu_{\mathbf{s}}^{\prime}\right)$, and the fiber diameter $\left(d_{\text {fiber }}\right)$, and is given as

$$
\left\langle\mathbf{L}_{\mathrm{SF}}\right\rangle=\frac{1.34 d_{\text {fiber }} \exp \left(0.17 d_{\text {fiber }}\right)}{\left(\mu_{\mathrm{s}}^{\prime} d_{\text {fiber }}\right)^{0.23}\left[0.52+\left(\mu_{\mathrm{a}}^{\text {tissue }} d_{\text {fiber }}\right)^{0.52}\right]} .
$$

This model structure requires specification of $\mu_{\mathrm{s}}^{\prime}$ at one wavelength, for which we choose $\mu_{s}^{\prime}(800 \mathrm{~nm})=1 \mathrm{~mm}^{-1}$, and the background scattering model estimates the wavelengthdependent change in $\mu_{\mathrm{s}}^{\prime}$; the effect of this assumption on model estimates is considered in Sec. 4.

Inspection of the data showed that absorption from an unknown chromophore was present within the SF reflectance spectra. Absorption from this unknown chromophore was accounted for by fitting absorption of an uncharacterized chromophore $\left(\mu_{\mathrm{a}}^{\text {unknown }}\right)$ with a Gaussian curve; a procedure that was described previously. ${ }^{13}$ This fitting technique estimated the wavelength of the absorption maximum for $\mu_{\mathbf{a}}^{\text {unknown }}$, which was similar to that of bilirubin as observed in vivo. ${ }^{14}$ The observation of bilirubin in measurements of in vivo tissue is reasonable because it is an endogenous compound that is a heme breakdown product and is found bound to albumin in human serum. Absorption from albumin-bound bilirubin within the SF spectra was considered by incorporating the contribution of bilirubin into Eq. (2), as

$$
\mu_{\mathrm{a}}^{\mathrm{tissue}}=\mu_{\mathrm{a}}^{\mathrm{vasc}}+\mu_{\mathrm{a}, \mathrm{spec}}^{\mathrm{bil}-\mathrm{l}} C_{\mathrm{bil}-\mathrm{al}} \text {. }
$$

Incorporation of Eq. (4) into Eq. (1) allowed the model to estimate the albumin-bound bilirubin concentration $\left(C_{\text {bil-al }}\right)$.

Model analysis of measured SF spectra yielded estimates of $\mathrm{StO}_{2}, \rho, d_{v}, C_{\text {bil-al }}$, and Mie slope $\left(a_{2}\right)$. The Mie amplitude $\left(a_{1}\right)$ was not compared between measurements on different nodes because the value of $a_{1}$ was dependent on the specific distance between the probe and spectralon during calibration measurements, a factor that was not uniformly controlled for all probes used in this study. Parameter estimation was achieved using a Levenberg-Marquardt algorithm to minimize the chi-squared metric between measured reflectance data and model predictions, and confidence intervals on parameter estimates were calculated from the square root of the diagonal of the covariance matrix. ${ }^{13}$ Statistical comparison of parameters estimated in normal and metastatic nodes was performed using a nonparametric Wilcoxon rank sum test, with significance determined by calculated $p$ values. In this study, spectra 
that showed evidence of a blood pool within the detection volume were identified as $\rho>40 \%$ and excluded from the comparative analysis.

\subsection{Bilirubin-Specific Absorption Coefficient}

Bilirubin, human serum albumin (HSA), and 99\% dimethyl sulfoxide (DMSO) were obtained from Sigma-Aldrich (Zwijndrecht, The Netherlands). Phosphate-buffered saline (PBS) was purchased from Gibco-Invitrogen (DH Breda, the Netherlands). Two stock solutions of bilirubin (concentrations: 0.2 and $3 \mathrm{mM}$ ) were prepared by dissolving bilirubin in DMSO and the solutions were stored in the dark at $4{ }^{\circ} \mathrm{C}$ until use. Stock solution of HSA (concentration: $0.25 \mathrm{mM}$ ) was prepared in PBS ( $\mathrm{pH}$ 7.4). The solutions of bilirubin-HSA complexes were prepared by mixing the stock solutions of bilirubin and HSA in PBS $(\mathrm{pH}=7.4)$. The final concentration of HSA in complexes was kept constant $(1 \mu \mathrm{M})$ and the concentration of bilirubin was varied between 0.25 and $20 \mu \mathrm{M}$. Complexes containing bilirubin concentrations in the range 0.25 to $3 \mu \mathrm{M}$ were prepared with the $0.2-\mathrm{mM}$ bilirubin stock solution, while complexes containing bilirubin concentrations in the range 5 to $20 \mu \mathrm{M}$ were prepared with the $3-\mathrm{mM}$ bilirubin stock solution. The bilirubin-HSA complexes prepared in PBS had a maximum residual content of DMSO of 1.5\%; this percentage is too low to have an effect on the complexes within solution. After preparation, the bilirubin-HSA complexes were stored in the dark in a water bath at $37{ }^{\circ} \mathrm{C}$ for $12 \mathrm{~h}$ to enable a complete equilibrium of the drug with the albumin. The bilirubin/HSA binding kinetics are known to be temperature dependent, ${ }^{15}$ so the $37{ }^{\circ} \mathrm{C}$ temperature was chosen to mimic the biological conditions in vivo.

Absorption spectra were recorded using a quartz cuvette $(1 \times 1 \mathrm{~cm})$ in an UV-VIS spectrophotometer (UV-2101 PC, Schimadzu Deutschland GmbH, Duisburg, Germany) in the wavelength range 240 to $800 \mathrm{~nm}$ with a slit width combination that resulted in a spectral resolution of $0.5 \mathrm{~nm}$. A distinct shift of the absorption maximum of bilirubin to a longer wavelength position was observed when bound to HSA, compared with bilirubin free (unbound) in the PBS. Therefore, it was important to consider the amount of bound and unbound bilirubin within the solutions, a factor that depended on the bilirubin/HSA concentration ratio. No changes in the wavelength position of the absorption maximum were observed for solutions with bilirubin concentrations $\leqslant 1 \mu \mathrm{M}$, a concentration that corresponds to a bilirubin/HSA ratio of $1: 1$. For molar ratios of bilirubin/HSA higher than 1:1, a spectral shift to shorter wavelength was observed that indicates saturation of the HSA binding sites and presence of free, unbound bilirubin molecules within the solution. This procedure enabled the determination of the specific absorption coefficient of bilirubin bound to HSA $\left(\mu_{a, \text { spec }}^{\text {bil-al }}\right)$.

\section{Results}

\subsection{Mathematical Model of $\mathbf{R}_{\mathrm{SF}}$ in Lymph Tissue In Vivo}

Figure 3(A) shows a representative single fiber reflectance $\left(\mathbf{R}_{\mathrm{SF}}\right)$ spectrum from the first (metastatic) lymph node sampled in the pilot study. We measured $\mathbf{R}_{\mathrm{SF}}$ at a resolution of 3 pixels $/ \mathrm{nm}$, and the data were smoothed by averaging data
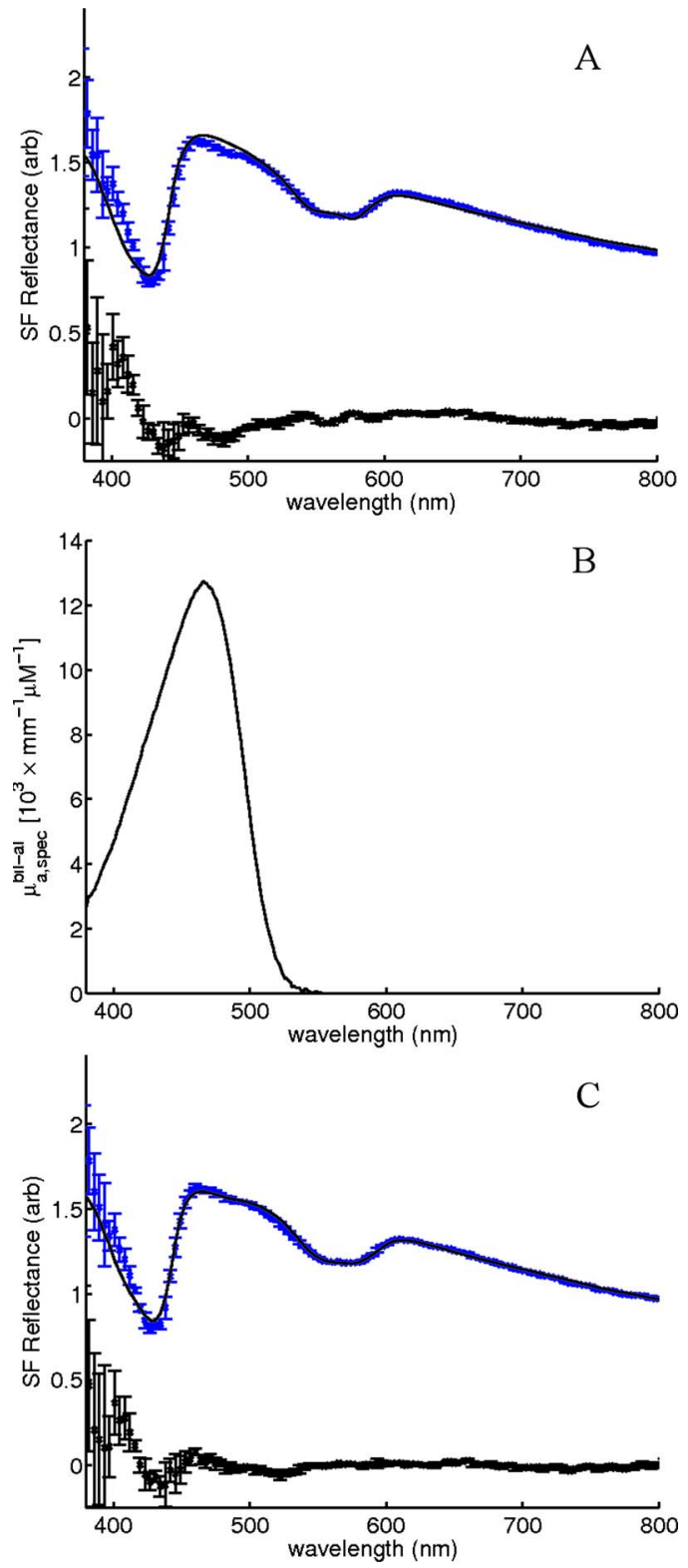

Fig. 3 SF reflectance model fit and $2 \times$ the residual with (A) absorption attributed to oxygenated and deoxyenated hemoglobin, (B) $\mu_{\mathbf{a} \text {,spec }}^{\text {bilal }}$ as measured in this study, and (C) model fit with bilirubin included in the basis set of chromophores.

points into bins of 10 pixels, which enabled the calculation of a standard deviation that represents noise within the signal. ${ }^{11}$ The line through the data set shows the mathematical model of the spectra (with absorption attributable to oxygenated and deoxygenated hemoglobin) and the dashed line below shows $2 \times$ the residual error (=data-model predictions). This model provided estimates of $\mathrm{StO}_{2}(30.3 \pm 3.7 \%), \rho(1.5 \pm 0.1 \%), d_{v}$ $(29.0 \pm 4.0 \mu \mathrm{m})$, and Mie slope $(-1.159 \pm 0.041)$. The Rayleigh amplitude estimate approached zero, indicating that this spectrum could be described by only Mie scattering (a finding that was confirmed in measurements on subsequent nodes). Although the fit in Fig. 3(A) seems reasonable, a detailed inspection of the residual error shows deviation between the model fit and the data in the 400- to 500-nm wavelength 


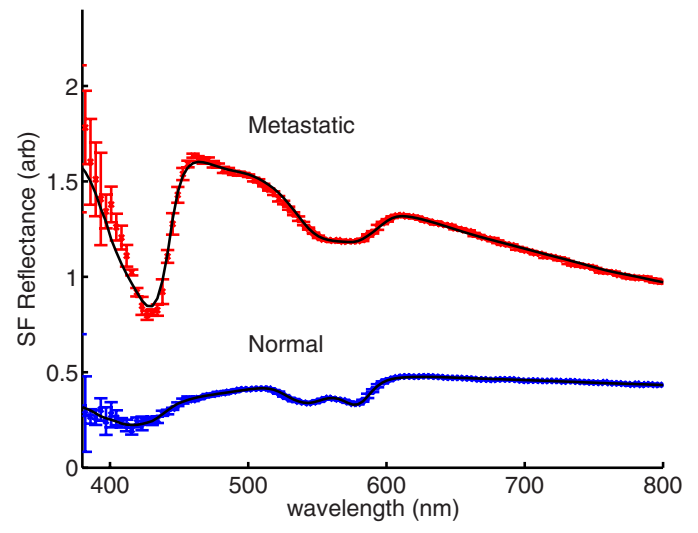

Fig. 4 Representative SF spectra and model fits from measurements on normal and metastatic lymph nodes, as confirmed by cytological examination.

range. Deviation in the 400- to $450-\mathrm{nm}$ range is attributable to the Soret band of hemoglobin, an issue that was described in detail previously. ${ }^{16}$ Deviations in the 450 - to 500 -nm range are attributed to improper specification of the basis set of chromophores, an issue that can have a profound effect on the estimated parameters. ${ }^{13}$ Therefore, we followed a systematic procedure to account for the absorption of an uncharacterized chromophore $\left(\mu_{\mathrm{a}}^{\text {unknown }}\right)$ with a Gaussian curve. Inclusion of the Gaussian resulted in a model fit that no longer showed the distinct deviation in the 450- to 500-nm wavelength range (data not shown). Interestingly, the Gaussian predicted an absorption maximum at $470 \mathrm{~nm}$, an observation that suggested bilirubin as the potential unknown absorber.

Figure 3(B) shows the specific absorption coefficient of albumin-bound bilirubin as measured in this study (with an absorption maximum of $0.0127 \mathrm{~mm}^{-1} \mu \mathrm{M}^{-1}$ at $466 \mathrm{~nm}$ ). The $\mathbf{R}_{\mathrm{SF}}$ model was reconfigured with bilirubin added as a chromophore contributing to the total tissue absorption coefficient; model predictions are shown in Fig. 3(C). The resulting model fit showed excellent agreement with the data and reduced the mean residual error between model and data by $64 \%$. The model with bilirubin added to the chromophore set provides an estimate of $C_{\text {bil-al }}(15.2 \pm 2.0 \mu \mathrm{M})$ as well as different parameter estimates for $\mathrm{StO}_{2}(11.8 \pm 1.8 \%), \rho(1.9 \pm 0.1 \%)$, and $d_{v}(50.1 \pm 4.1 \mu \mathrm{m})$ compared with estimates from the model fit without bilirubin; other parameters did not change by more than $10 \%$. These observations suggest that bilirubin contributes meaningfully to the total absorption detected within the lymph tissue in vivo, and that incorporation of bilirubin into the basis set of chromophores influences the estimated values of the other model parameters. As a result, bilirubin concentration was added to the set of physiological parameters extracted from the SF measurements in lymph tissue.

\subsection{Model Analysis of Clinical Pilot Data}

Figure 4 shows representative $\mathbf{R}_{\mathrm{SF}}$ spectra from both normal and metastatic lymph nodes, and the line through each data set shows the mathematical model for each respective spectrum. These spectra show differences that are attributable to the differences in tissue composition and morphology. Parameters estimated by the fitted mathematical model indicated
Table 1 Mean physiological and morphological parameters extracted from SF reflectance measurements of normal and metastatic lymph nodes in vivo.

\begin{tabular}{lcc}
\hline Parameter & $\begin{array}{c}\text { Normal } \\
(7 \text { nodes })\end{array}$ & $\begin{array}{c}\text { Metastatic } \\
(8 \text { nodes })\end{array}$ \\
\hline Hemoglobin saturation $(\%)$ & $83.9 \pm 10.0$ & $46.8 \pm 24.9^{a}$ \\
Blood volume fraction $(\%)$ & $13.5 \pm 7.6$ & $3.2 \pm 2.6^{a}$ \\
Average vessel diameter $(\mu \mathrm{m})$ & $13.9 \pm 10.9$ & $8.9 \pm 5.0$ \\
Bilirubin $(\mu \mathrm{M})$ & $23.2 \pm 12.4$ & $10.2 \pm 5.5$ \\
Mie slope & $-0.71 \pm 0.34$ & $-1.13 \pm 0.38$ \\
\hline Indicates significant differences with $p<0.01$ for the Kruskal Wallis test.
\end{tabular}

major differences between these two representative spectra in $\mathrm{StO}_{2}(85.9 \pm 2.3$ versus $11.9 \pm 1.8 \%), \rho(7.6 \pm 0.2 \%$ versus $2.0 \pm 0.1 \%)$, and $d_{v}(20.8 \pm 17.0$ versus $50.0 \pm 4.1 \mu \mathrm{m})$ for the normal and metastatic nodes, respectively. Data for all nodes measured in the clinical pilot study are shown in Table 1 , which displays the mean values for each of the five parameter factors for all spectra measured in both normal and metastatic nodes. Significant differences between normal and metastatic nodes were found in $\mathrm{StO}_{2}$ and $\rho$ (with $p$ values of $<0.01$ ). Differences in the mean values of $d_{v}, C_{\text {bil-al }}$, and Mie slope were not significant. These results indicate that physiological differences between normal and metastatic nodes could be detected by the SF reflectance measurement.

\subsection{Effect of SF Probe on Lymph Node Environment}

To observe the effect that manipulation of the SF probe has on the lymph node environment, we performed rapid measurements over a time frame with pressure applied to the probe, maintained for a time interval, and then released. Figure 5 shows temporal profiles of $\mathrm{StO}_{2}$ and $\rho$ as measured in one normal [Fig. 5(A)] and one metastatic [Fig. 5(B)] lymph node. The $x$ axis of each plot provides the time with respect to the onset of the application of pressure. The data for time $<0 \mathrm{~s}$ show measurements with the probe placed into the lymph node, but no additional pressure being applied. Horizontal dashed lines indicate the onset of pressure (at time $=0 \mathrm{~s}$ ) and the subsequent release of pressure at $26 \mathrm{~s}$ in Fig. 5(A) and at $22 \mathrm{~s}$ in Fig. 5(B). The data in both plots show decreases in both $\mathrm{StO}_{2}$ and $\rho$ as pressure is applied to the probe and maintained; this temporal functionality was also observed for $d_{v}$ (data not shown). These observations showed a distinct effect of pressure on physiology, and are attributed to the pressure compressing the tissue surrounding the probe tip, which impairs blood flow to the local vasculature. Pressure-induced changes to morphology (scattering) were not considered to be substantial $(<20 \%)$, and showed no temporal functionality. The data in the plot in Fig. 5(B) also show dramatic increases in the volume of blood sampled by the probe after the release of probe pressure; an observation that indicates the onset of bleeding and formation of a blood pool. These results indicate that the pressure exerted by the SF probe can have major effects on the physiological environment of the lymph node being sampled. 

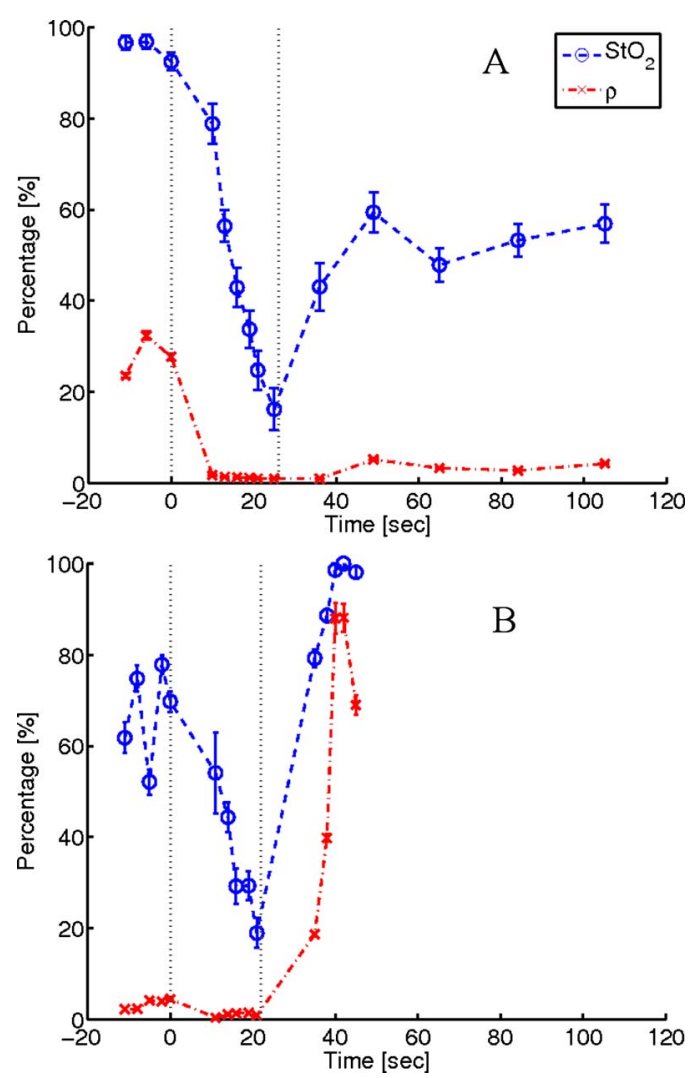

Fig. 5 Effect of single fiber probe pressure on physiological environment, with changes observed in hemoglobin saturation and blood volume fraction over time for one (A) normal and one (B) metastatic lymph node. The $x$ axis shows time with respect to the onset of pressure by the probe on the lymph tissue. Horizontal dashed lines indicate the onset and release of pressure.

\section{Discussion}

This study shows the feasibility and presents the technical aspects of incorporating an SF reflectance spectroscopy device into the EUS-FNA technique for lung cancer staging in vivo. Preliminary clinical data are used to develop an analytical model of $\mathbf{R}_{\mathrm{SF}}$ measured in lymph tissue in vivo. A systematic mathematical methodology is used to characterize the basis set of chromophores in lymph tissue using a Gaussian curve. ${ }^{13}$ The estimated absorption maximum for the Gaussian was similar to that of bilirubin as observed in vivo. ${ }^{14}$ However, previous studies have utilized a specific absorption coefficient of bilirubin as measured in chloroform for analysis of in vivo spectra, ${ }^{17}$ a problem because the absorption maximum of bilirubin in chloroform is ${ }^{18}$ at $\approx 453 \mathrm{~nm}$, while bilirubin bound to serum albumin shows an absorption maximum at $\approx 466 \mathrm{~nm}$. Our attempts to utilize the specific absorption coefficient of bilirubin as measured in chloroform yielded poor model fits that showed observable features within the residual (data not shown). It is well-known that the specific absorption coefficient of a compound depends on the surrounding matrix; this motivated measurement of bilirubin absorption in an environment that mimics the in vivo situation, i.e., in the presence of albumin. Incorporation of the albumin-bound bilirubin spectra into the model resulted in a high-quality fit, with a large reduction in the mean error about the model and with an observable improvement in the residual plot. Physiological parameter estimates were also affected by the inclusion of bilirubin in the model. Specifically, we observed changes in $\mathrm{StO}_{2}(30.3$ versus $11.8 \%), \rho$ (1.5 versus $\left.1.9 \%\right)$, and $d_{v}(29.0$ versus $50.1 \mu \mathrm{m}$ ), as calculated by the model without and with bilirubin incorporated, respectively. These results highlight the impact that improper specification of the in vivo chromophore set can have on estimates of model parameters. ${ }^{13}$ While this study does not obtain independent confirmation of the presence of bilirubin within the sampled lymph nodes, the data strongly suggest that bilirubin is an important source of absorption within lymph tissue in vivo. Definitive confirmation of these results would require analysis of the bilirubin content within the tissue extracted during the EUS-FNA procedure; while this is beyond the scope of the current study, it could be incorporated into a future protocol.

Analysis of $\mathbf{R}_{\mathrm{SF}}$ spectra measured in this clinical pilot study were capable of detecting differences in the physiology between normal and metastatic lymph nodes. The data shown in Table 1 indicate that metastatic nodes presented lower $\mathrm{StO}_{2}$ and lower $\rho$ than normal nodes. Previous studies that investigated the vascular supply of metastatic lymph nodes reported the presence of aberrant vessels, displacement of vessels, and avascular areas, with the primary source of perfusion from the peripheral vasculature. ${ }^{19,20}$ In advanced stages of metastatic lymph nodes, the regular vascular network throughout the center of the lymph node may be destroyed or displaced due to the tumor cells, a problem that can be exacerbated by central necrosis or keratinization within the node. ${ }^{21}$ These observations are consistent with the description of a compromised vascular network that provides an explanation of the decreases in $\mathrm{StO}_{2}$ and $\rho$ observed in metastatic lymph nodes compared with normal nodes within the current study. Interestingly, studies of the physiological environment of tumors in the breast, lung, and oral cavity ${ }^{6-8}$ show reduced $\mathrm{StO}_{2}$ and increased $\rho$ compared with normal functioning tissue in each case. The difference in changes to $\rho$ between normal and tumor tissue and normal and metastatic lymph nodes can be described by the difference in the respective local environments. Solid tumors have an increased $\rho$ compared with normal tissue because the tumor cells up-regulate angiogenesis, increasing blood supply to meet the needs of highly proliferating cells. ${ }^{22}$ In metastatic lymph nodes, neoplastic cells infiltrate the node from outside and are usually located in the peripheral areas; therefore, when the metastatic site(s) upregulate angiogenesis, the effect is not uniform throughout the node and results in increased peripheral vascularization. ${ }^{21}$ The subsequent proliferation of the metastatic site(s) in the peripheral areas of the node exacts a mechanical pressure to the center of the node, compressing blood vessels and increasing the resistance to blood flow, ${ }^{23}$ factors that result in a reduction in $\rho$ (and $\mathrm{StO}_{2}$ ) within the node.

It is important to put the results associated with the comparison of the physiology between normal and metastatic lymph nodes into the proper clinical context. The EUS-FNA is utilized in lung cancer staging to provide definitive confirmation about the presence of metastatic cancer within lymph nodes that appear enlarged on a CT scan. The current study compares the tissue parameters of normal (not enlarged) lymph nodes with metastatic (enlarged) lymph nodes; how- 
ever, it is the discrimination between benign nodes that are enlarged due to infection or nonmalignant disease and nodes enlarged due to presence of metastatic regions that is the clinically relevant question. Previously, it has been reported that lymph nodes enlarged due to immune response present vessel dilation and hypervascularity, with a relationship between perfusion and volume of the enlarged nodes. ${ }^{24}$ These observations suggest that differences in the vascular networks between benign enlarged and metastatic lymph nodes may translate into differences in physiology that are detectable by the SF reflectance measurement. However, this hypothesis has yet to be tested experimentally and will be addressed by a future study.

The authors anticipate the need to address some specific technical challenges prior to widespread clinical acceptance of the combined EUS-FNA and SF spectroscopy technique. One such concern is the lack of a relationship between the SF reflectance intensity and $\mu_{\mathrm{s}}^{\prime}$. The spectral analysis algorithm presented in this paper requires an assumption of $\mu_{\mathrm{s}}^{\prime}$ at a single wavelength [we chose $\mu_{s}^{\prime}(800 \mathrm{~nm})=1 \mathrm{~mm}^{-1}$ ] and the wavelength-dependent changes in scattering across the spectra are described by a background scattering model. The error introduced to parameter estimates due to this assumption was quantified by varying the assumed $\mu_{s}^{\prime}(800 \mathrm{~nm})$ across a wide range that is representative of biological tissue $\left(0.5-2.0 \mathrm{~mm}^{-1}\right)$, with resulting changes to the estimates of $\rho$, $\mathrm{StO}_{2}, d_{v}$, and Mie slope of $20 \%,<1 \%, 14 \%$, and $<1 \%$, respectively. While these results indicate the error is small, it is desirable to not require the assumption within the model, so we are currently developing a stable optical phantom that can be used to calibrate a measured SF reflectance intensity to a value of $\mu_{s}^{\prime}$.

Another technical challenge is avoiding the perturbation of the lymph tissue in vivo environment with the optical probe during sampling. The sampling strategy described in Fig. 1 involves manipulation of the SF probe throughout the lymph node being sampled in an attempt to characterize a larger volume of the node than is achieved by the current procedure. Manipulation of the probe to different locations within the node requires the application of pressure to the probe tip. However, the data in Fig. 5 indicate that the application of pressure can compress the surrounding tissue and induce major changes in the tissue physiology; specifically to the microvasculature. This is problematic because pressure applied to a normal node can result in reduced $\rho$ and $\mathrm{StO}_{2}$, factors that were associated with metastatic nodes; this phenomenon is displayed in Fig. 5(A). Moreover, the pressure effects do not introduce any unique characteristics into the measured spectra, making it difficult to develop a system to provide online identification and correction. Note that pressureinduced changes in scattering were not substantial and showed no time dependence. Another concern associated with probe manipulation is tissue damage that results in bleeding. This can lead to the optical detection of a blood pool instead of native tissue, as observed in Fig. 5(B). However, this is not expected to be a serious problem for the technique, because the presence of blood pools can be identified by establishing a threshold blood volume fraction (as was performed in this study). In this study, the authors utilized a sampling strategy that is expected to minimize the effect of pressure on the measurements with spectra acquired in the following manner: the EUS-FNA probe was inserted into the node, the SF probe extended and was allowed to rest with no external pressure being applied during the SF measurement. Therefore, while we have confidence that these pressure effects did not influence the data set presented in Table 1, the implementation of the sampling strategy outlined in Fig. 1 may prove to be infeasible.

In conclusion, this paper showed the successful incorporation of the EUS-FNA with incorporated SF reflectance spectroscopy as demonstrated in the clinical theater. A mathematical model was developed to extract physiological and morphological parameters from the SF reflectance spectra measured in lymph nodes in vivo; analysis included incorporation of albumin-bound bilirubin into the basis set of chromophores. Model analysis was performed on spectra measured in a clinical pilot study and results indicate that the SF measurement is sensitive to differences in tissue physiology between normal and metastatic lymph nodes. Moreover, clinical observations show that probe pressure can have major effects on the physiological environment of the lymph node; effects that must carefully be considered when developing a sampling strategy. The data presented in this study show the feasibility of this novel technique, however, the clinical utility of this technology will be based on the ability to assist in the classification of enlarged lymph nodes as either benign or metastatic; a question that will be investigated in future studies.

\section{Acknowledgments}

This research is supported by the Dutch Technology Foundation STW, the applied science division of Nederlandse Organisatie voor Wetenschappelijk Onderzoek (NWO), the Technology Program of the Ministry of Economic Affairs, and the Maurits and Anna de Kock Foundation.

\section{References}

1. U.S. Cancer Statistics Working Group, Department of Health and Human Services, Centers for Disease Control and Prevention, and National Cancer Institute (2007).

2. F. C. Detterbeck, M. M. DeCamp Jr., L. J. Kohman, and G. A. Silvestri, "Invasive staging: the guidelines," Chest 123, 167S-175 (2003).

3. G. Caddy, M. Conron, G. Wright, P. Desmond, D. Hart, and R. Y. Chen, "The accuracy of EUS-FNA in assessing mediastinal lymphadenopathy and staging patients with NSCLC," Eur. Respir. J. 25, 410 415 (2005)

4. M. A. Eloubeidi, A. Tamhane, V. K. Chen, and R. J. Cerfolio, "Endoscopic ultrasound-guided fine needle aspiration of mediastinal lymph node in patients with suspected lung cancer after positron emission tomography and computed tomography scans," Ann. Thorac. Surg. 80, 1231-1239 (2005).

5. D. T. Delpy and M. Cope, "Quantification in tissue near-infrared spectroscopy," Philos. Trans. R. Soc. London, Ser. B 352, 649-659 (1997).

6. R. L. P. van Veen, A. Amelink, M. Menke-Pluymers, C. van der Pol, and H. J. C. M. Sterenborg, "Optical biopsy of breast tissue using differential path-length spectroscopy," Phys. Med. Biol. 50, 25732581 (2005).

7. M. P. L. Bard, A. Amelink, V. Noordhoek Hegt, W. J. Graveland, H. J. C. M.. Sterenborg, H. C. Hoogsteden, and J. G. J. V. Aerts, "Measurement of hypoxia-related parameters in bronchial mucosa by use of optical spectroscopy," Am. J. Respir. Crit. Care Med. 171, 11781184 (2005).

8. A. Amelink, O. P. Kaspers, H. J. C. M. Sterenborg, J. E. vander Wal, J. L. N. Roodenburg, and M. J. H. Witjes, "Non-invasive measure- 
ment of the morphology and physiology of oral mucosa by use of optical spectroscopy," Oral Oncol. 44, 65-71 (2008).

9. T. P. Moffit and S. A. Prahl, "In-vivo sized-fiber spectroscopy," Proc. SPIE 3917, 225-231 (2000).

10. M. Canpolat and J. R. Mourant, "Particle size analysis of turbid media with a single optical fiber in contact with the medium to deliver and detect white light," Appl. Opt. 40, 3792-3799 (2001).

11. S. C. Kanick, H. J. C. M. Sterenborg, and A. Amelink, "Empirical model of the photon path length for a single fiber reflectance spectroscopy device," Opt. Express 17, 860-871 (2009).

12. R. Reif, M. S. Amorosino, K. W. Calabro, O. A'Amar, S. K. Singh, and I. J. Bigio, "Analysis of changes in reflectance measurements on biological tissues subjected to different probe pressures," J. Biomed. Opt. 13, 010502 (2008).

13. A. Amelink, D. J. Robinson, and H. J. C. M. Sterenborg, "Confidence intervals on fit parameters derived from optical reflectance spectroscopy measurements," J. Biomed. Opt. 13, 054044 (2008).

14. S. L. Jacques, I. Saidi, A. Ladner, and D. Oelberg, "Developing an optical fiber reflectance spectrometer to monitor bilirubinemia in neonates," Proc. SPIE 2975, 115-127 (1997).

15. R. Brodersen, "Bilirubin. Solubility and interaction with albumin and phospholipid," J. Biol. Chem. 254(7), 2364-2369 (1979).

16. A. Amelink, T. Christiaanse, and H. J. C. M. Sterenborg, "Effect of hemoglobin extinction spectra on optical spectroscopic measurements of blood oxygen saturation," Opt. Lett. 34, 1525-1527 (2009).

17. L. Lyngsnes Randeberg, E. Bruzell Roll, L. T. Norvang Nilsen, T. Christensen, and L. O. Svaasand, "In vivo spectroscopy of jaundiced newborn skin reveals more than a bilirubin index," Acta Paediatr. 94, 65-71 (2005).

18. H. Du, R. A. Fuh, J. Li, A. Corkan, and J. S. Lindsey, "PhotochemCAD: a computer-aided design and research tool in photochemistry," Photochem. Photobiol. 68, 141-142 (1998).

19. A. Tschammler, H. Wirkner, G. Ott, and D. Hahn, "Vascular patterns in reactive and malignant lymphadenopathy," Eur. Radiol. 6, 473 480 (1996).

20. T. Chikui, K. Yuasa, S. Maemura, and S. Kanda, "Change of angiostructure and hemodynamics in lymph node metastases in rabbits," Oral Surg. Oral Med. Oral Pathol. Oral Radiol. Endod. 93, 350-357 (2002).

21. M. Ying and A. T. Ahuja, "Ultrasound of neck lymph nodes: how to do it and how do they look?" Radiology 12, 105-117 (2006).

22. R. K. Jain, "Determinants of tumor blood flow: a review," Cancer Res. 48, 2641-2658 (1988).

23. A. T. Ahuja and M. Ying, "Sonographic evaluation of cervical lymph nodes," AJR, Am. J. Roentgenol. 184, 1691-1699 (2005).

24. P. G. Herman, D. Lyonnet, R. Fingerhut, and R. N. Tuttle, "Regional blood flow to the lymph node during the immune response," Lymphology 9, 101-104 (1976). 